\title{
Ultrasonic Wave Propagation Through Aberrating Layers: Experimental Verification of the Conjugate Gradient Rayleigh Method
}

\author{
Léon A. F. Ledoux, Arthur P. Berkhoff, Student Member, IEEE, and Johan M. Thijssen, Senior Member, IEEE
}

\begin{abstract}
The Conjugate Gradient Rayleigh method for the calculation of acoustic reflection and transmission at a rough interface between two media was experimentally verified. The method is based on a continuous version of the conjugate gradient technique and plane-wave expansions. We measured the beam profiles of a nonfocused linear array transducer distorted by propagation through an aberrating interface. The directivity of the hydrophone was compensated for by means of spatial deconvolution. Sinusoidal, cylindrical aberrators were made of a water-based gelatine material. The measurements were compared with 2- and 3-D simulations. The measured and simulated beam profiles are in good agreement, as shown by the root-mean-square difference.
\end{abstract}

\section{INTRODUCTION}

$\mathbf{I}$ $\mathrm{N}$ echographic imaging, an important distortion of the ultrasonic beams is caused by aberrations. Aberrations are phase and amplitude errors, which are often caused by irregular interfaces between tissue layers with a different propagation speed.

In some cases, these aberrations can be compensated for by use of an array transducer. The elements of such a transducer can be excited following a certain correction algorithm [1]-[4]. In order to investigate the applicability and limits of aberration correction methods, it is important to have a reliable and efficient numerical model of the propagation of sound passing through rough interfaces. Berkhoff et al. presented in [5] the acoustic Conjugate Gradient Rayleigh (CGR) method, which is an iterative method for the calculation of acoustic reffection and transmission at a rough interface between two media. The iterative method is based on a continuous version of the conjugate gradient technique, where the field variables are expanded in a sum (or integral) of outgoing plane waves.

A comparison of the method with a rigorous integral equation method was presented in [6]. Although the CGR method is not as accurate as the integral equation method, it is accurate enough for the application in which we are interested. For the present application, the method is much more efficient than integral equation methods or methods using direct matrix

Manuscript received March 28, 1995; revised September 8, 1995. This work was supported by a grant from Philips Medical Systems B.V., Best, The Netherlands and by a grant from the Technological Science Branch of the Dutch Organization for Scientific Research, Project NGN 11.2427.

The authors are with the Biophysics Laboratory, Department of Ophthalmology, St. Radboud University Hospital, $6500 \mathrm{HB}$ Nijmegen, The Netherlands.

Publisher Item Identifier S 0885-3010(96)00371-1.

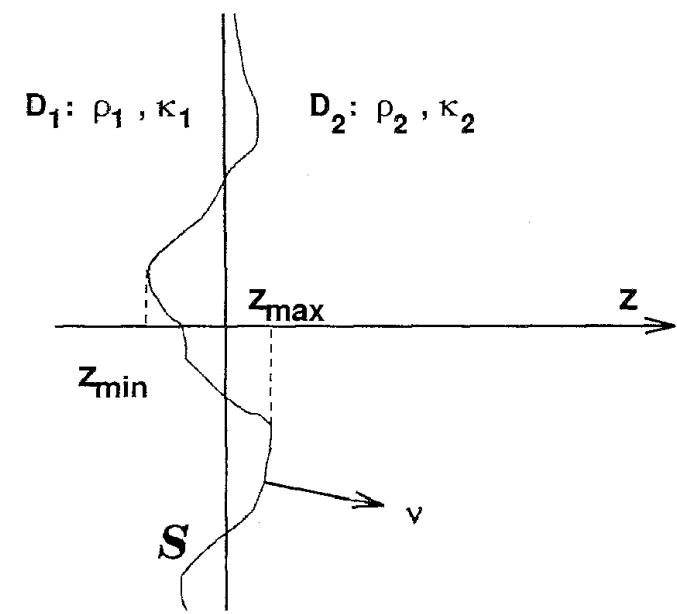

Fig. 1. Two-dimensional definition of the different domains in the configuration.

inversion. Our application deals with surfaces of moderate roughness and low velocity contrast between the media. Then, the error of the algorithm is sufficiently small. It should be noted that the error of the algorithm (on the rough interface) is available in the iteration scheme. The error in the pressure field away from the interface is of comparable size, but smaller than the error on the rough interface. Therefore, we have always available an upper bound of the CGR simulation error.

In this paper, the CGR method is experimentally verified. The simulations based on the CGR method are compared with measurements in which artificial aberrators have been used.

\section{THEORY}

\section{A. Summary of the Conjugate Gradient Rayleigh Method}

It is assumed that the roughness of the interface is a local deformation of an otherwise plane boundary at $z=0$, where a point in space is specified by its right-handed, orthogonal coordinates $x, y, z$ (see Fig. 1). The analysis is carried out in the temporal frequency domain with angular frequency $\omega$ where the complex time factor $e^{-i \omega t}$ is suppressed in the following. The two fluid-like media occupy the domains $\mathcal{D}_{1}$ and $\mathcal{D}_{2}$, respectively, and are assumed to be linear, homogeneous, and isotropic with respective mass densities $\rho_{1}$ 


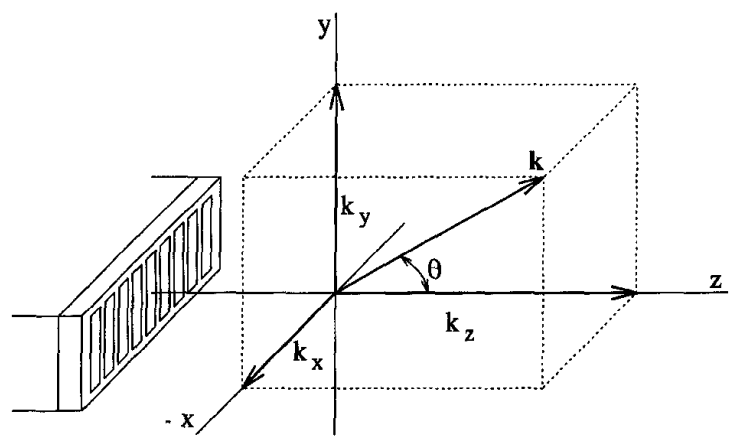

Fig. 2. Orientation of the transducer and the $\mathbf{k}$-vector corresponding to the direction of the transmitted plane waves.

and $\rho_{2}$ and compressibilities $\kappa_{1}$ and $\kappa_{2}$. Furthermore, both media exhibit some losses and the real and imaginary parts of $\rho$ and $\kappa$ satisfy the Kramers-Kronig causality relations. The interface is denoted by $\mathcal{S}$, and the pressure and the particle velocity vector by $P$ and $\mathbf{V}$, respectively. In $\mathcal{D}_{1}$, a source of finite extent generates a wave incident upon $\mathcal{S}$. The incident wave is denoted by $\left\{P_{i}, \mathbf{V}_{i}\right\}$. The total field in $\mathcal{D}_{1}$ is written as the superposition of the incident field and the reflected field $\left\{P_{r}, \mathbf{V}_{r}\right\}$. The total field in $\mathcal{D}_{2}$ is the transmitted field $\left\{P_{t}, \mathbf{V}_{t}\right\}$.

The solution of the reffection/transmission problem is found by expanding the field in a sum (or integral) of plane waves [5]. The plane wave coefficients are obtained by an iterative minimization of the integrated squared error in the boundary conditions at the rough interface. We assume the existence of an iterative procedure, in which $n$ steps have been carried out. The iterative procedure has led to the plane-wave components $\phi_{r}^{(n)}$ and $\phi_{t}^{(n)}$ of the reflected and transmitted velocity potentials, respectively. The corresponding field values are

$$
\begin{aligned}
& \left\{P_{r}^{(n)}, \mathbf{V}_{r}^{(n)}\right\}=\iint_{-\infty}^{\infty}\left\{p_{r}^{(n)}, \mathbf{v}_{r}^{(n)}\right\} e^{i \mathbf{k}_{1}^{-} \cdot \mathbf{x}} d k_{x} d k_{y}, \mathbf{x} \in \mathcal{D}_{1}, \\
& \left\{P_{t}^{(n)}, \mathbf{V}_{t}^{(n)}\right\}=\iint_{-\infty}^{\infty}\left\{p_{t}^{(n)}, \mathbf{v}_{t}^{(n)}\right\} e^{i \mathbf{k}_{2}^{+} \cdot \mathbf{x}} d k_{x} d k_{y}, \mathbf{x} \in \mathcal{D}_{2},
\end{aligned}
$$

where

$$
\begin{aligned}
& \left\{p_{r}^{(n)}, \mathbf{v}_{r}^{(n)}\right\}=i\left\{\omega \rho_{1}, \mathbf{k}_{1}^{-}\right\} \phi_{r}^{(n)} \\
& \left\{p_{t}^{(n)}, \mathbf{v}_{t}^{(n)}\right\}=i\left\{\omega \rho_{2}, \mathbf{k}_{2}^{+}\right\} \phi_{t}^{(n)}
\end{aligned}
$$

The plane-wave vectors $\mathbf{k}_{1}^{-}$and $\mathbf{k}_{2}^{+}$indicate plane waves traveling away from $\mathcal{S}$

$$
\mathbf{k}_{1}^{-}=\left(k_{x}, k_{y},-k_{z, 1}\right) \text {, }
$$

with

$$
k_{z, 1}=\left(\omega^{2} \rho_{1} \kappa_{1}-k_{x}^{2}-k_{y}^{2}\right)^{1 / 2} \Re\left(k_{z, 1}\right), \Im\left(k_{z, 1}\right)>0,
$$

and

$$
\mathbf{k}_{2}^{+}=\left(k_{x}, k_{y},+k_{z, 2}\right),
$$

with

$$
k_{z, 2}=\left(\omega^{2} \rho_{2} \kappa_{2}-k_{x}^{2}-k_{y}^{2}\right)^{1 / 2} \Re\left(k_{z, 2}\right), \Im\left(k_{z, 2}\right)>0 .
$$

At the interface $\mathcal{S}$ the fields should satisfy the boundary conditions

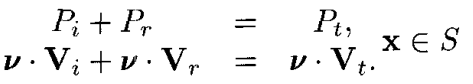

In the Appendix, we show how we can construct $\phi_{r, t}^{(n)}$ in such a way that the error in the approximation of the boundary conditions is reduced at each iteration step. Using a Cauchy-Schwarz inequality, it was shown in [5] that the use of an integrated squared criterion in combination with the outgoing plane wave representations (1) leads to a valid solution of the reflection/transmission problem.

\section{B. 2.5-D Algorithm}

If the interface of the aberrator is constant in one direction, here the $y$-direction, then some computational savings can be made. The method is as follows: first, the incident wave field is Fourier-transformed in the $y$-direction (see Fig. 2). The result is that the sound field is expressed as a sum of cylindrical wave fields, each with a corresponding value of $k_{y}$. The $2-$ $\mathrm{D}$ version of the iterative algorithm is applied to each of the cylindrical wave fields. For the cylindrical wave fields, the $y$-position only induces a multiplicative term representing a phase-shift, so we can take $y=0$ without loss of generality. After applying the complete 2-D iterative algorithm for each value of $k_{y}$, the desired 3-D reflected and transmitted fields are obtained by an inverse Fourier transform in the $y$-direction. Note that the terms $k_{y} y$ and $k_{y} \nu_{y}$ disappear [(17), (18), (21), and (22)], but the terms with $k_{z, 1}$ and $k_{z, 2}$ still contain a $k_{y}$-component. We will call this version of the algorithm the 2.5-D algorithm.

\section{Transducer Model}

For reasons to be explained later, we decided to model the transducer. The transducer model is based on the following methods. The multi-element transducer is assumed to have a uniform normal surface velocity for each element. Cross coupling between elements has been neglected because the level was less than $-40 \mathrm{~dB}$ relative to the main pulse. The conversion of transducer velocity to a spatial sampling grid is obtained by linear interpolation, as in [7]. The resulting normal velocity distribution in the transducer plane is transformed (by fast Fourier transform) to the wave number domain. The sound field extrapolation from the transducer plane to the rough interface results from the use of a weak form of a kspace Green's function similar to the methods of [8] and [9]. From the plane-wave decomposition of the field incident on the rough surface, we can apply equations similar to (1) to calculate the incident field $\left\{P_{i}, \mathbf{V}_{i}\right\}$. Time-domain results can be obtained by analyzing the problem at several frequencies and subsequently calculating the inverse (temporal) Fourier transform. 


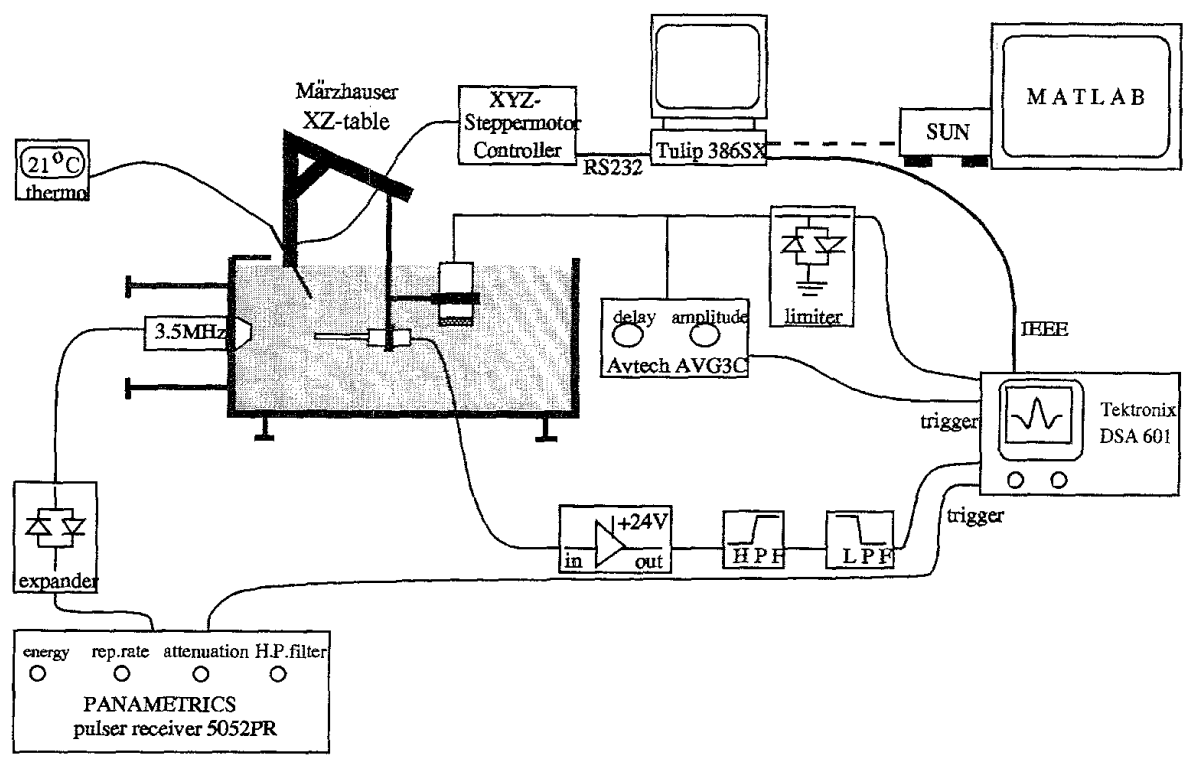

Fig. 3. Schematic diagram of the equipment.

\section{EXPERIMENTAL METHODS}

\section{A. Experimental Arrangement}

The overall experimental arrangement for the measurement of the ultrasonic fields is shown in Fig. 3. The acoustical pressure transmitted by an electrically nonfocused linear array transducer with 128 elements (pitch: $0.76 \mathrm{~mm}$, height: 18 $\mathrm{mm}$ ) and a center frequency of $3.5 \mathrm{MHz}$ was sensed by a needle hydrophone. The hydrophone was manufactured at the Delft University of Technology. The hydrophone has a built-in amplifier with a gain of $21.5 \mathrm{~dB}$. The hydrophone signal was digitized by a Tektronix DSA601 digitizing signal analyzer. To get an improvement of the signal-to-noise ratio, the signal was band-limited with a first-order high-pass filter having a cutoff frequency of approximately $500 \mathrm{kHz}$, and a fourth-order Bessel low-pass filter having a cutoff frequency of $15 \mathrm{MHz}$. The signal-to-noise ratio was further improved by averaging every signal 16 times.

A Märzhauser $X Z$-table, which was controlled by an $X Y Z$-steppermotor controller, was employed for positioning the hydrophone in the region of interest. The $X Z$-table was able to make steps of $1 \mu \mathrm{m}$. A Tulip 386SX microcomputer was the heart of the data acquisition for storage of the data as well as for control of the hydrophone positioning during the measurement. The data stored on the microcomputer's hard disk were transmitted to a SUN-workstation where they were processed using Matlab ${ }^{\mathrm{TM}}$ version 4.2.

The transducer was fixed in one side of a perspex water tank in such a way that the $X Z$-plane of the transducer was parallel to the bottom of the water tank. (This has been verified by performing some measurements in the $X Z$-plane of the transducer at two opposite positions of the $Y$-axis. The profiles of these two $X Z$-scans were almost the same, which is the case when the $X Z$-plane of the transducer is parallel to the scanning plane and thus to the bottom of the water tank.) To avoid interference due to echoes reflected from the walls of the water tank, the dimensions of the tank were taken much larger than the dimensions of the acoustic fields to be measured. Furthermore, the rate of the pulser was kept low. The hydrophone was placed in the water tank via a construction that was connected to the $X Z$-table. The arm movement between the $X Z$-table and the hydrophone was not critical because the measurements were performed in socalled "start-stop" mode. In this way, the hydrophone vibration during the data-acquisition process was minimized.

The array transducer was driven by a short electronic pulse (80-ns duration) with a $140-\mathrm{V}$ peak voltage. This pulse was generated by a Panametrics 5052PR pulser-receiver that was used as transmitter only. An expander was interfaced between the transducer and the pulser to disconnect the pulser from the circuit during the receive period to eliminate noise from the pulser electronics [10].

We found that the accuracy of the measurements depended for a great part on the alignment of the array transducer with respect to the scanning-plane of the hydrophone. Therefore, we added a single-element transducer to our measurement system. This transducer was mounted on the arm connected to the hydrophone. The transducer was directed perpendicular to the bottom of the water tank, which was parallel to the $x z$-plane of the array transducer (see Fig. 2). The transducer was excited by an Avtech AVG-3-C pulser. During the transmit part, the oscilloscope was protected from the high source voltage by means of a limiter. The transducer was moved, successively, to the four corners of the $x z$-scan region. The maximum of the reflected echo waveform sensed by the transducer had to be located at the same instant of time for all the four corners. If this was not the case, then the height of the water tank had to be altered.

The measurements were performed at room temperature, typically $21^{\circ} \mathrm{C}$. Care was taken that during the measurement 


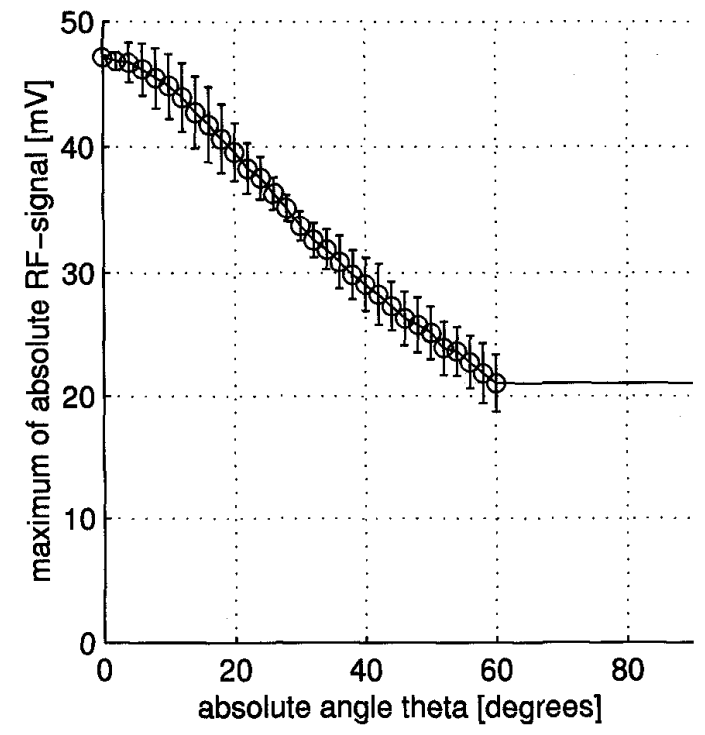

Fig. 4. The mean and standard deviation of the directivity of the hydrophone.

the temperature fluctuations of the water were within reasonable margins.

\section{B. Compensation for the Hydrophone Directivity}

Hydrophones are typically used to measure the local pressure within ultrasonic fields. The active element of our needle hydrophone was located in the end of a small cylindrical housing, resulting in minimal perturbation of the ultrasonic field. The hydrophone was made of a PVDF polymer, with a sensitive surface of $0.25 \mathrm{~mm}^{2}$.

To reduce the influence of the hydrophone, we compensated for its directivity. The directivity was measured at a frequency of $3.5 \mathrm{MHz}$, where the hydrophone was placed in the focus of a continuous wave excited single element transducer. The angle $\theta$ between the normal on the transducer surface and the normal on the hydrophone surface was changed $\left(0^{\circ}\right.$ corresponds to the transducer position in line with the hydrophone). The maximum of the absolute RF signal was determined for each of the positions.

The directivity measurement was performed for two perpendicular planes. The graph of the mean directivity $d(\theta)$ with its standard deviation is shown in Fig. 4. The standard deviation is a measure of the degree of rotational symmetry of the hydrophone directivity. Based on this graph, we assumed the directivity to be rotationally symmetric and to be equal to the mean value of the measured data.

The directivity could only be measured for angles $\theta$ between $-60^{\circ}$ and $60^{\circ}$. For larger absolute angles, we took the value at $60^{\circ}$. This has the advantage that side lobes with low signalto-noise ratios get limited compensation.

The directivity function can be transformed to the spatial frequency domain using the following relation (see Fig. 2):

$$
\theta=\arcsin \left(\frac{\sqrt{k_{x}^{2}+k_{y}^{2}}}{k}\right),
$$

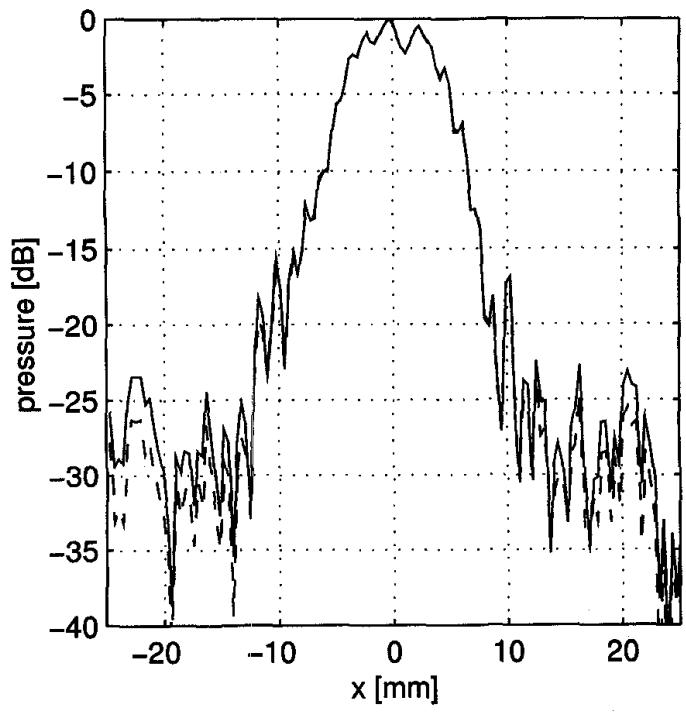

Fig. 5. Monochromatic beam profile showing the effect of hydrophone compensation. Dashed: without compensation. Solid: with compensation.

where

$$
k=\left(k_{x}^{2}+k_{y}^{2}+k_{z}^{2}\right)^{1 / 2} .
$$

The influence of the hydrophone directivity was removed by means of a spatial deconvolution process. This can be formulated as

$$
p_{t r}\left(k_{x}, k_{y}\right)=D^{-1}\left(k_{x}, k_{y}\right) p_{m}\left(k_{x}, k_{y}\right),
$$

where $p_{t r}\left(k_{x}, k_{y}\right)$ is the spatial Fourier transform of the true field, $p_{m}\left(k_{x}, k_{y}\right)$ is the spatial Fourier transform of the measured field, and $D\left(k_{x}, k_{y}\right)$ is the spatial Fourier transform corresponding with the hydrophone directivity. Taking the inverse Fourier transform of $p_{t r}$ resulted in the desired spatial field.

An example of the result of the compensation method is shown in Fig. 5. This figure shows the amplitude of the 3.5-MHz frequency component of the RF signals of an $x$ scan (step-size $0.38 \mathrm{~mm}$ ) made at a $z$-distance of $30 \mathrm{~mm}$ for the case in which 14 elements were excited. The dashed line corresponds to the measured signal without compensation and the solid line corresponds to the measured signal with compensation.

\section{Aberrators}

The aberrators were constructed in such a way that a reasonable approximation to the surface roughness and medium properties found in human tissue was obtained.

The sound speed of the aberrator medium has an important impact on the aberration of the sound field, so the material should have a sound speed between $1450 \mathrm{~ms}^{-1}$ and $1700 \mathrm{~ms}^{-1}$ to be a close approximation of human tissue. To get the desired sound speed, we used gelatine as tissue mimicking material. Gelatine solutions are made by dissolving a prescribed mass concentration of gelatine powder in water (at $70^{\circ} \mathrm{C}$ ). 


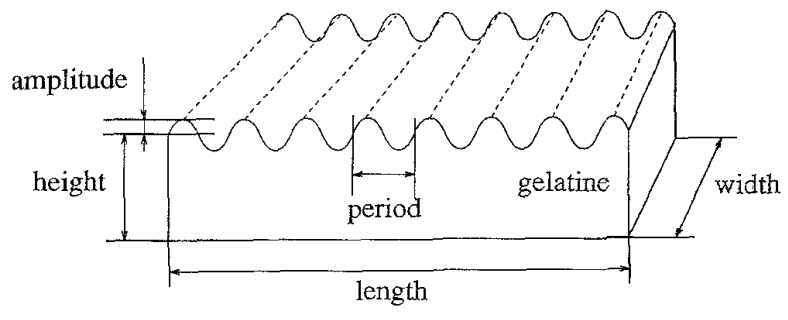

Fig. 6. Dimensions of gelatine aberrator.

TABLE I

Characteristics of the Gelatine Aberrators

\begin{tabular}{|c|c|c|c|c|c|c|c|}
\hline $\begin{array}{c}\text { type of } \\
\text { aberrator }\end{array}$ & $\begin{array}{c}\text { percentage } \\
\text { gelative }\end{array}$ & $\begin{array}{c}\text { period } \\
{[\mathrm{mm}]}\end{array}$ & $\begin{array}{c}\text { amplitude } \\
{[\mathrm{mm}]}\end{array}$ & $\begin{array}{c}\text { height } \\
{[\mathrm{mm}]}\end{array}$ & $\begin{array}{c}\text { sound apeed } \\
{\left[\mathrm{mg}^{-1}\right]}\end{array}$ & $\begin{array}{c}\text { attenuation } \\
{\left[\mathrm{dBcm}^{-1} \mathrm{MBz}^{-1}\right]}\end{array}$ & $\begin{array}{c}\text { density } \\
{\left[\mathrm{gcm}^{-3}\right]}\end{array}$ \\
\hline aberrator 1 & $20 \%$ & 3 & 0.5 & 35 & 1550 & 0.12 & 1.20 \\
\hline aberrator 2 & $25 \%$ & 3 & 0.5 & 28 & 1600 & 0.20 & 1.25 \\
\hline aberrator 9 & $25 \%$ & 4 & 0.5 & 10 & 1600 & 0.20 & 1.25 \\
\hline
\end{tabular}

The aberrators were made with a flat surface on one side and on the opposite side a 2-D irregular surface, i.e., the surface was constant in one direction.

We used two different molds for the construction of the aberrators. Both molds had a sinusoidal surface. This sinusoidal surface made it easier to determine the exact position of the aberrator in front of the transducer because of the periodic behavior of the sinusoidal surface.

The sinusoidal surface is characterized by its amplitude and period (see Fig. 6). We used an amplitude of $0.5 \mathrm{~mm}$ (peak-peak $1 \mathrm{~mm}$ ), which is a realistic representation of the very irregularly and wavy border between the dermis and the subcutaneous fat layer [11]. The smallest period that practically could be manufactured having this amplitude was a period of $3 \mathrm{~mm}$. We made one mold with a period of $3 \mathrm{~mm}$ and one with a period of $4 \mathrm{~mm}$.

The length and width of the molds were not critical, as long as they covered the complete region of the transducer surface corresponding to the excited elements.

We made three different gelatine aberrators, aberrator 1 , aberrator 2 , and aberrator 3 , respectively. The characteristics of these aberrators are listed in Table I.

\section{RESULTS}

\section{A. 2-D Versus 3-D and 2.5-D Simulations}

The simulations could be performed in 2- and 3-D. In the 2-D simulations, the height of the elements was considered to be infinite.

Simulations in 2-D, where only the $k_{y}=0$ term is used, are not as accurate as the 3-D simulations. The reason for this can be seen in Fig. 7, where the waveforms are shown as a function of $x$ for $y=0$ at a depth of $z=30 \mathrm{~mm}$ for a 14 element array in a homogeneous medium. We can see that the $3-\mathrm{D}$ simulations include the edge waves caused by the finite transducer size in the $y$-direction (see the region between 21 and $21.5 \mu \mathrm{s}$ ).

Fig. 8 compares the monochromatic ( $3.5 \mathrm{MHz}$ ) beam profiles of a 2- and 2.5-D simulation with the corresponding measurement. These beam profiles correspond to an $x$-scan of

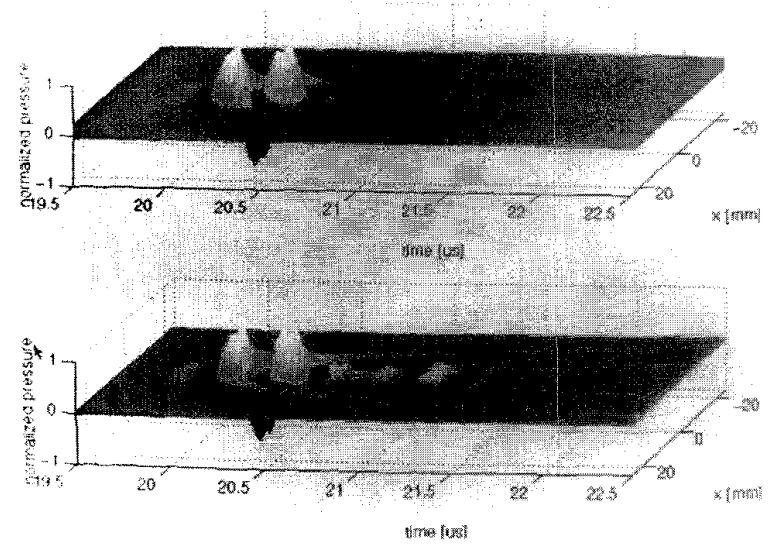

Fig. 7. Two-dimensional plane wave simulation versus 3-D plane wave simulation (14 elements at $z=30 \mathrm{~mm}$ ). The RF signals are plotted on a linear grayscale.

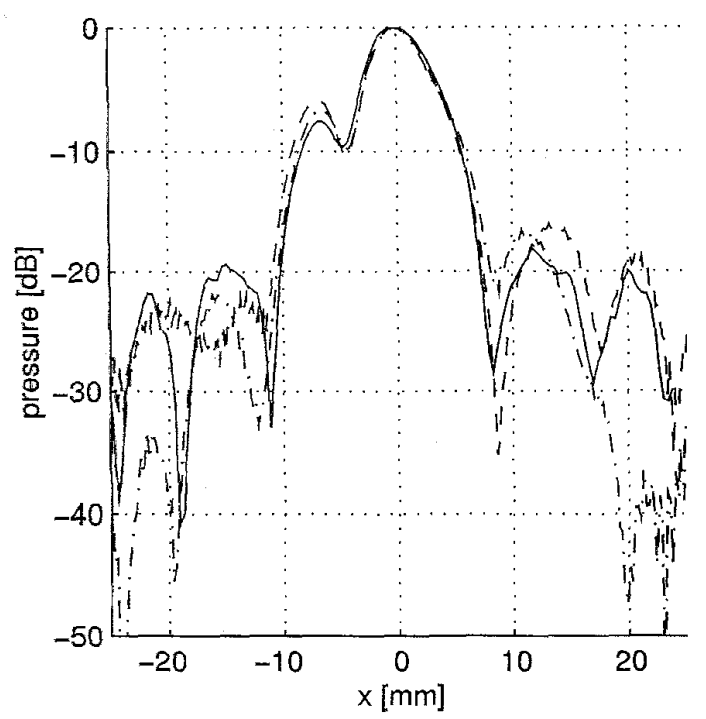

Fig. 8. Measurement (solid line) compared with 2-D CGR simulation (dash-dotted line) and 2.5-D CGR simulation (dashed line).

eight excited elements at a depth of $80 \mathrm{~mm}$. Aberrator 3 was placed in front of the transducer (the flat side of the aberrator was placed against the surface of the transducer). The figure shows that the 2.5-D simulation is more accurate, i.e., closer to the results of the measurement, than the 2-D simulation, especially in the regions beside the main lobe. The signals in these regions are for the greater part caused by edge waves. The beam profile of the 2-D simulation omits the edge waves caused by the finite height of the elements, and therefore the signal is much lower than that of the $2.5-\mathrm{D}$ simulations in the region beside the main lobe. In the following, we used 2.5-D simulations instead of the much faster 2-D simulations.

\section{B. Verification of the Transducer Model}

The CGR algorithm requires the field $\left\{P_{i}, V_{i}\right\}$ incident at the interface as input, as can be seen in (14). There are several possibilities for obtaining this incident field. 


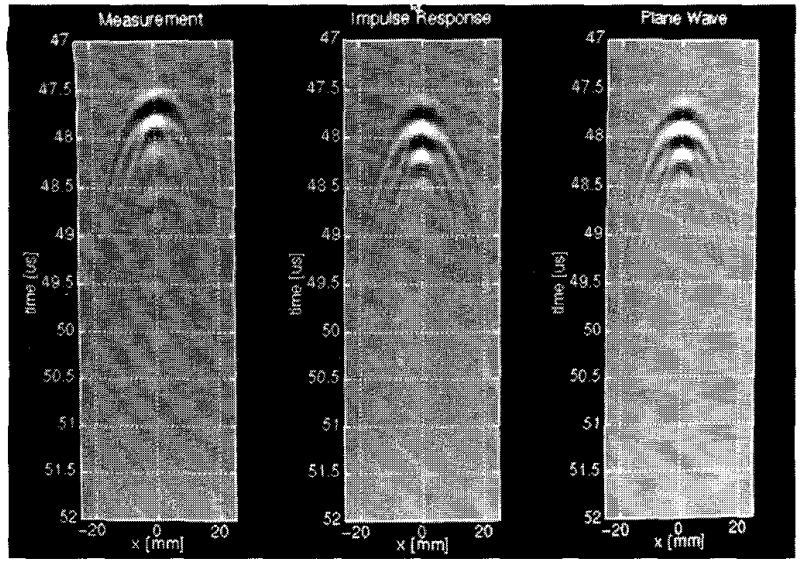

Fig. 9. $x$-scans of 18 elements at $100 \mathrm{~mm}$.

One method is to measure the field at the location of the aberrator surface, where the aberrator is removed. This method is not very attractive because the hydrophone has to follow exactly the aberrating surface.

Another possibility is to measure the field in a plane near the aberrator followed by calculation of $\left\{P_{i}, \mathbf{V}_{i}\right\}$ at the rough surface. This method was not feasible because of the large amount of time needed to scan such a plane properly.

A third method of obtaining the incident field $\left\{P_{i}, \mathbf{V}_{i}\right\}$ is to model the transducer, which is the method we have used. To verify the correctness of the transducer model, some scans were made with only water in the measurement tank. Scans were made in the $x$-direction at $y=0$. The measurements were performed for several combinations of the number of excited elements and the distance between the transducer and the hydrophone. The combinations were selected more or less randomly, but in such a way that they would be representative for scan conditions that could occur during the measurements with the aberrators.

The measured and simulated $x t$-plots showed good agreement. The simulations were performed with two methods, the plane wave decomposition method [12] and the impulse response method [13]. The transducer pulse was in both simulation methods assumed to be a Gaussian pulse with a central frequency of $3.5 \mathrm{MHz}$ and a $-6-\mathrm{dB}$ bandwidth of 2.0 MHz. An example of these $x t$-plots is shown in Fig. 9. The plots correspond to $x$-scans of eight excited elements at a depth of $70 \mathrm{~mm}$.

To get a better comparison between the simulations and measurements, we determined the frequency component of the RF signals corresponding to the central frequency of the array transducer, i.e., $3.5 \mathrm{MHz}$. An example of the results of this method is shown in Fig. 10. This graph corresponds to the $x t$-plots shown in Fig. 9. The solid line represents the measurement, the dashed line represents the plane wave decomposition simulation, and the dashed-dotted line corresponds to the impulse response method.

The following equation has been used as a quantitative measure for the comparison of the simulations and measurements. The equation is based on the root-mean-square

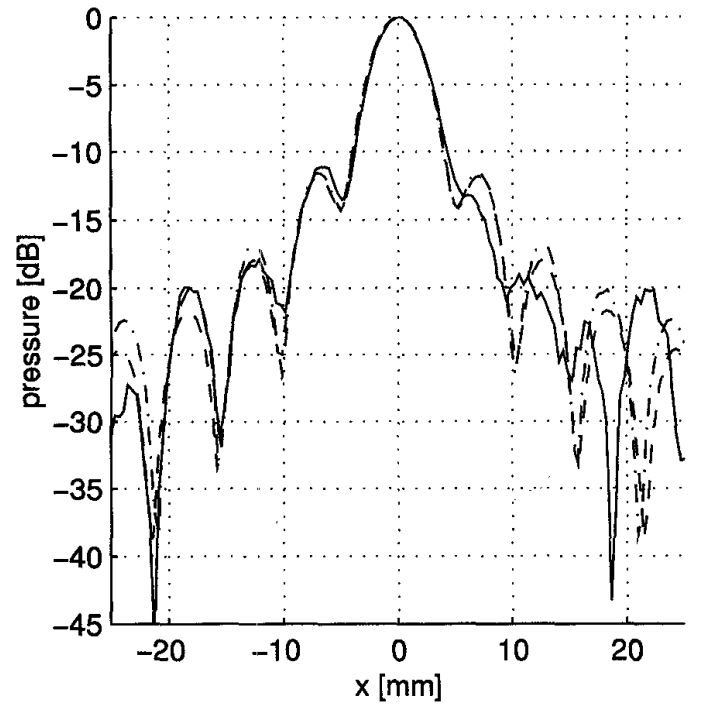

Fig. 10. Comparison of the profiles corresponding to the $x$-scans of 18 elements at $100 \mathrm{~mm}$ (solid line: measurements; dashed line: simulations).

TABLE II

Comparison of Measurements and Simulations of the Transducer (an asterisk Means That the Measurement Has not Been Performed)

\begin{tabular}{|c|c|c|c|c|}
\hline \multirow{2}{*}{$\begin{array}{c}\text { number of } \\
\text { elements }\end{array}$} & \multirow{2}{*}{$\begin{array}{c}\text { simulation } \\
\text { method }\end{array}$} & \multicolumn{3}{|c|}{ comp } \\
\cline { 3 - 6 } & $\mathrm{z}=30 \mathrm{~mm}, \mathrm{~m}$ & $\mathrm{z}=50 \mathrm{~mm}$ & $\mathrm{z}=70 \mathrm{~mm}$ \\
\hline \hline 8 & plane wave decomposition & 0.183 & 0.137 & 0.083 \\
\cline { 2 - 6 } & impulise response & 0.221 & 0.148 & 0.090 \\
\hline \multirow{2}{*}{14} & plane wave decomposition & 0.134 & 0.108 & $*$ \\
\cline { 2 - 6 } & impulse response & 0.141 & 0.115 & $*$ \\
\hline
\end{tabular}

difference comprms between the measured and simulated $x t$ scan using the values corresponding to the $3.5-\mathrm{MHz}$ frequency component. This resulted in the following:

$$
\text { comp }_{\mathrm{rms}}=\sqrt{\frac{\sum_{x=1}^{N_{x}}\left|v_{m}(x)-v_{\operatorname{sim}}(x)\right|^{2}}{\sum_{x=1}^{N_{x}}\left|v_{m}(x)\right|^{2}}}
$$

where $N_{x}$ is the number of scan-points in the $x$-direction, $v_{m}(x)$ is the absolute normalized voltage of the measurement at position $x$ for the $3.5-\mathrm{MHz}$ frequency component, and $v_{s i m}(x)$ is the same as $v_{m}(x)$, but in this case the normalized pressure values of the simulation (either the plane wave decomposition method or the impulse response method) must be used.

Some of the results of this comparison method are listed in Table II (an asterisk means that the measurement has not been performed). These results show that both simulation methods give a realistic representation of the wavefront transmitted by the array transducer.

\section{Comparison of Measured and Simulated Aberrations}

For the verification of the CGR method, several $x$-scans were made with aberrators in front of the transducer. The (cylindrical) surface of the aberrator was constant in the $y$ direction. The flat side of the aberrator was placed against the surface of the transducer. The hydrophone was positioned in 


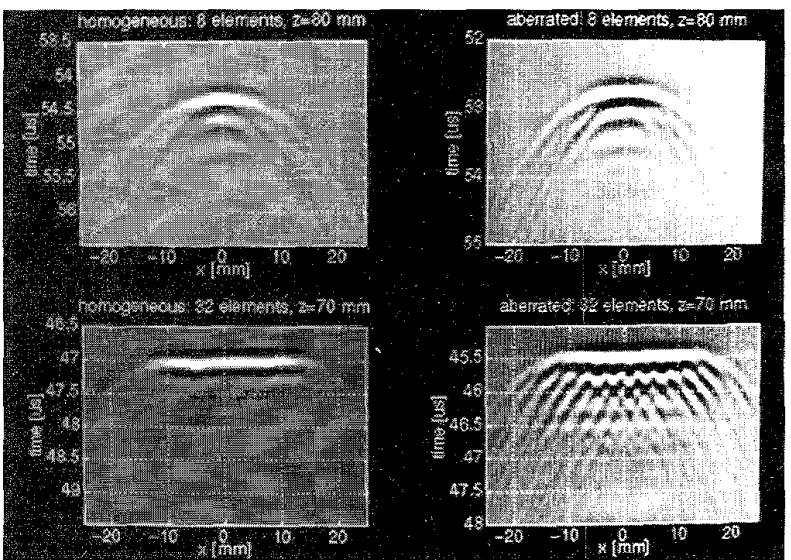

Fig. 11. Effects of aberrator 2 on $x$-scans. Top: $x$-scans of eight excited elements at $z=80 \mathrm{~mm}$. Bottom: $x$-scans of 32 excited elements at $z=$ $70 \mathrm{~mm}$. Left: measurements performed in water. Right: measurements with aberrator 2 in front of the transducer.

the middle of the $y$-direction of the elements during the measurements. We varied the number of elements and the distance between the transducer and the hydrophone. Furthermore, we used three types of aberrators.

Fig. 11 compares the effects of homogeneous and aberrating media. The upper graphs correspond to an $x$-scan with eight excited elements at a $z$-distance of $80 \mathrm{~mm}$, and the graphs underneath correspond to an $x$-scan with 32 excited elements at a $z$-distance of $70 \mathrm{~mm}$. In both cases, the left graph corresponds to an $x$-scan in a homogeneous medium, i.e., water, while the right graph corresponds to an $x$-scan with aberrator 2 in front of the transducer.

Several $x$-scans (step-size $0.38 \mathrm{~mm}$, i.e., half of the pitch) were made for different configurations. All these $x$-scans have been simulated. The CGR simulations made use of the following settings. We used 512 interface points, i.e., the number of plane waves, in the $x$-direction and in the $y$ direction. The evanescent modes were omitted. The simulation step sizes in the $x$-direction and in the $y$-direction were equal to $0.4 \lambda$ where $\lambda=\min \left(\lambda_{\text {water }}, \lambda_{\text {aberrator }}\right)$. Because of the rapid convergence of the algorithm, only one iteration was required. More iterations did not lead to significantly better results.

The simulation error is defined as the root-mean-square error normalized to the case of vanishing reflected and transmitted fields, i.e.,

$$
\widehat{E R R}^{(n)}=\left(\frac{E R R^{(n)}}{E R R_{0}}\right)^{1 / 2}
$$

where $E R R^{(n)}$ is the integrated squared error in the boundary conditions after $n$ steps of iteration [see (13)]. The value of $E R R_{0}$ is the initial value $E R R^{(0)}$ where the plane wave components $\phi_{r}^{(0)}$ and $\phi_{t}^{(0)}$ are zero. The simulation error $\widehat{E R R}^{(n)}$ for $n=1$ was approximately 0.13 .

The position of the aberrator in front of the transducer was determined by altering the position of the aberrator (steps as small as $0.01 \mathrm{~mm}$ were necessary) in the simulation until the resemblance between the measurement and simulation was
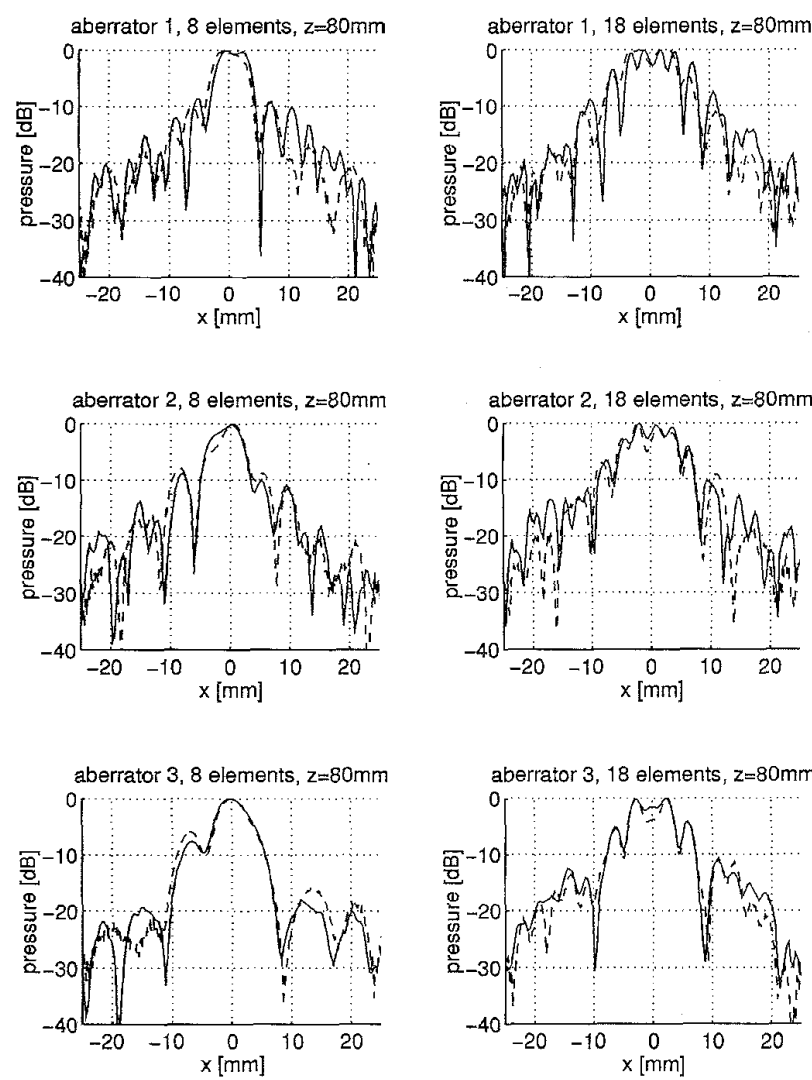

Fig. 12. Comparison between the monochromatic ( $3.5 \mathrm{MHz}$ ) beam profiles of measurements (solid line) and of 2.5-D CGR simulations (dashed line). Left: eight excited elements at $z=80 \mathrm{~mm}$. Right: 18 excited elements at $z=80 \mathrm{~mm}$. Top: aberrator 1. Middle: aberrator 2. Bottom: aberrator 3 .

as close as possible. For these position-determining simulations, the much faster 2-D algorithm was used, but the final simulations were performed in 2.5-D.

Fig. 12 gives an overview of several monochromatic (3.5 $\mathrm{MHz}$ ) beam profiles of two different $x$-scans that were performed for the three aberrators. The solid line corresponds to the measurement and the dashed line corresponds to the 2.5-D simulation. The left graphs correspond to eight excited elements at a $z$-distance of $80 \mathrm{~mm}$ and the right graphs correspond to 18 excited elements at a $z$-distance of $80 \mathrm{~mm}$. The upper part corresponds to aberrator 1 , the middle part to aberrator 2 , and the lower part to aberrator 3 . It is remarkable that the graphs of the measurements have sharper dips than the graphs of the simulations.

Again, (11) has been used to compare the results of the simulations and the measurements. Some of the results are listed in Table III (an asterisk means that the measurement has not been performed).

Comparing the data in Table III with those in Table II reveals that the results of the simulations of aberrators are only slightly worse than the simulations of the homogeneous media. This is an indication that the CGR method actually might be more accurate than the results of Table III suggest. The difference between Tables II and III can be explained as follows. Firstly, the simulation method introduces an error 
TABLE III

Comparison of Measurements and Simulations with ABerrators (an asterisk Means That the Measurement Has not Been Performed)

\begin{tabular}{|c|c|c|c|c|}
\hline \multirow{2}{*}{$\begin{array}{c}\text { type of } \\
\text { aberrator }\end{array}$} & \multirow{2}{*}{$\begin{array}{l}\text { number of } \\
\text { elements }\end{array}$} & \multicolumn{3}{|c|}{ comp $_{R M S}$} \\
\hline & & $\mathrm{z}=70 \mathrm{~mm}$ & $\mathrm{z}=80 \mathrm{~mm}$ & $\mathrm{z}=90 \mathrm{~mm}$ \\
\hline \multirow[t]{2}{*}{ aberrator 1} & 8 & $\bar{*}$ & 0.234 & \\
\hline & 18 & ${ }^{*}$ & 0.282 & * \\
\hline \multirow{4}{*}{ aberrator 2} & 8 & * & $0 . \overline{83}$ & $*$ \\
\hline & 14 & 0.227 & 0.230 & 0.264 \\
\hline & 18 & 0.247 & 0.199 & 0.314 \\
\hline & 32 & 0.268 & $*$ & 0.288 \\
\hline \multirow[t]{2}{*}{ aberrator 3} & 8 & $*$ & $0 . \overline{115}$ & * \\
\hline & 18 & * & 0.132 & \\
\hline
\end{tabular}

because of the nonzero residual error $E R R^{(N)}$ if we truncate the calculation in iteration $N$. Secondly, we found that the position of the aberrator relative to the transducer was very critical. Finally, the acoustic properties of the aberrators, which were made of gelatine, slightly change as time progresses. In view of the relatively small differences between Tables II and III and the large impact of the aberrators on the beam cross sections (compare, for instance, the location and magnitude of the peaks and dips of Figs. 8 and 10), we can safely state that the measurements confirm the simulations with the CGR method.

\section{CONCLUSION}

The experimental verification showed that the simulations of the Conjugate Gradient Rayleigh method accurately predict the beam distortion due to an aberrating interface between two media.

Furthermore, it has been shown that 3- and 2.5-D simulations are more accurate than 2-D simulations in the regions beside the main lobe. This is caused by the fact that 2-D simulations omit the edge waves caused by the finite height of the elements.

\section{APPENDIX: CONJUGATE GRAdIENT RAYLEIGH METHOD [5]}

In the following, it is shown how the plane-wave components $\phi_{r}^{(n)}$ and $\phi_{t}^{(n)}$ of the reflected and transmitted velocity potentials can be constructed such that the error in the approximation of the boundary conditions is reduced in an iterative way.

The integrated squared error $E R R^{(n)}$ in the boundary conditions after $n$ steps of iteration is defined as

$$
E R R^{(n)}=\int_{\mathbf{x} \in \mathcal{S}}\left(\left|F_{P}^{(n)}\right|^{2}+\left|F_{V}^{(n)}\right|^{2}\right) d \mathbf{x},
$$

in which the deviations $F_{P}^{(n)}=F_{P}^{(n)}(\mathbf{x}), F_{V}^{(n)}=F_{V}^{(n)}(\mathbf{x})$ are given by

$$
\begin{aligned}
& F_{P}^{(n)}=Y_{0}^{1 / 2}\left(P_{i}+P_{r}^{(n)}-P_{t}^{(n)}\right) \\
& F_{V}^{(n)}=Z_{0}^{1 / 2} \boldsymbol{\nu} \cdot\left(\mathbf{V}_{i}+\mathbf{V}_{r}^{(n)}-\mathbf{V}_{t}^{(n)}\right)
\end{aligned}
$$

with $Z_{0}=\left(\kappa_{0} / \rho_{0}\right)^{1 / 2}$ and $Y_{0}=\left(\rho_{0} / \kappa_{0}\right)^{1 / 2}$ a reference impedance and admittance, and $\nu=\nu(x)$ the outward normal of the surface $\mathcal{S}$ at $\mathrm{x}$, pointing into $\mathcal{D}_{2}$.
In going from the $(n-1)$ st step to the $n$ th, we take

$$
\phi_{r, t}^{(n)}=\phi_{r, t}^{(n-1)}+\eta^{(n)} g_{r, t}^{(n)},
$$

where $\eta^{(n)}$ is a variational parameter to minimize $E R R^{(n)}$, and $g_{r, t}^{(n)}=g_{r, t}^{(n)}\left(k_{x}, k_{y}\right)$ are the search directions.

Combining the results of (1), (2), (14), and (15), it can be shown that

$$
F_{P, V}^{(n)}=F_{P, V}^{(n-1)}-\eta^{(n)} f_{P, V}^{(n)},
$$

in which

$$
\begin{aligned}
f_{P}^{(n)} & =-i \omega \rho_{1} Y_{0}^{1 / 2} \iint_{-\infty}^{\infty} g_{r}^{(n)} e^{i \mathbf{k}_{1}^{-} \cdot \mathbf{x}} d k_{x} d k_{y} \\
& +i \omega \rho_{2} Y_{0}^{1 / 2} \iint_{-\infty}^{\infty} g_{t}^{(n)} e^{i \mathbf{k}_{2}^{+} \cdot \mathbf{x}} d k_{x} d k_{y} \\
f_{V}^{(n)} & =-i Z_{0}^{1 / 2} \iint_{-\infty}^{\infty} \boldsymbol{\nu} \cdot \mathbf{k}_{1}^{-} g_{r}^{(n)} e^{i \mathbf{k}_{1}^{-} \cdot \mathbf{x}} d k_{x} d k_{y} \\
& +i Z_{0}^{1 / 2} \iint_{-\infty}^{\infty} \nu \cdot \mathbf{k}_{2}^{+} g_{t}^{(n)} e^{i \mathbf{k}_{2}^{+} \cdot \mathbf{x}} d k_{x} d k_{y}
\end{aligned}
$$

For the search directions, we can take the conjugate gradient directions [14]

$$
\begin{gathered}
g_{r, t}^{(n)}=s_{r, t}^{(n-1)}+\left(A^{(n)} / A^{(n-1)}\right) g_{r, t}^{(n-1)} n \geq 2 \\
g_{r, t}^{(1)}=s_{r, t}^{(0)},
\end{gathered}
$$

where the symbols $s_{r}^{(n)}$ and $s_{t}^{(n)}$ denote the steepest descent directions

$$
\begin{aligned}
s_{r}^{(n)}= & {\left[-i \omega \rho_{1} Y_{0}^{1 / 2} \int_{\mathbf{x} \in \mathcal{S}} F_{P}^{(n)} e^{i \mathbf{k}_{1}^{-} \cdot \mathbf{x}} d \mathbf{x}-i Z_{0}^{1 / 2}\right.} \\
& \left.\cdot \int_{\mathbf{x} \in \mathcal{S}} \nu \cdot \mathbf{k}_{1}^{-} F_{V}^{(n)} e^{i \mathbf{k}_{1}^{-} \cdot \mathbf{x}} d \mathbf{x}\right]^{*}, \\
s_{t}^{(n)}= & {\left[+i \omega \rho_{2} Y_{0}^{1 / 2} \int_{\mathbf{x} \in \mathcal{S}} F_{P}^{(n)} e^{i \mathbf{k}_{2}^{+} \cdot \mathbf{x}} d \mathbf{x}+i Z_{0}^{1 / 2}\right.} \\
& \left.\cdot \int_{\mathbf{x} \in \mathcal{S}} \boldsymbol{\nu}^{\prime \cdot} \cdot \mathbf{k}_{2}^{+} F_{V}^{(n)} e^{i \mathbf{k}_{2}^{+} \cdot \mathbf{x}} d \mathbf{x}\right]^{*},
\end{aligned}
$$

and where

$$
A^{(n)}=\iint_{-\infty}^{\infty}\left(\left|s_{r}^{(n-1)}\right|^{2}+\left|s_{t}^{(n-1)}\right|^{2}\right) d k_{x} d k_{y} .
$$

The variational paranneter $\eta^{(n)}$ resulting from the choice for the conjugate gradient directions is given by

$$
\eta^{(n)}=A^{(n)} / B^{(n)},
$$

with

$$
B^{(n)}=\int_{\mathbf{x} \in \mathcal{S}}\left(\left|f_{P}^{(n)}\right|^{2}+\left|f_{V}^{(n)}\right|^{2}\right) d \mathbf{x} .
$$

It can be proven [5] that the error satisfies

$$
E R R^{(n)}=E R R^{(n-1)}-\left|A^{(n)}\right|^{2} / B^{(n)} .
$$


As a result, we have a decrease of the error if $A^{(n)} \neq 0$. From (23), we can see that this is the case if $s_{r, t}^{(n-1)} \neq 0$. However, if $s_{r, t}^{(n-1)}$ vanishes, we have arrived at the exact solution in the iteration $n-1$.

\section{ACKNOWLEDGMENT}

The authors are grateful to P. Both of Philips Medical Systems B.V. for making available the array transducer, $G$. Faber of the laboratory for Seismics and Acoustics of the Delft University of Technology for supplying the hydrophone, R. Hekkenberg and W. Oosterbaan of the TNO Center for Medical Technology, Leiden, for the measurement of the hydrophone directivity, and M. Cuypers for his support during the development of the experimental setup. They would also like to thank Prof. P. M. van den Berg for his suggestions and remarks.

\section{REFERENCES}

[1] L. Nock, G. E. Trahey, and S. W. Smith, "Phase aberration correction in medical ultrasound using speckle brightness as a quality factor," $J$. Acoust. Soc. Amer., vol. 85, pp. 1819-1833, 1989.

[2] C. Gambetti and F. S. Foster, "Correction of phase aberrations for sectored annular array ultrasound transducers," Ultrasound Med. Biol., vol. 19, pp. $763-776,1993$.

[3] M. Karaman, A. Atalar, H. Kömen, and M. O'Donnell, "A phase aberration correction method for ultrasound imaging," IEEE Trans. Ultrason., Ferroelect., Freq. Contr., vol. 40, pp. 275-282, 1993.

[4] D. L. Liu and R. C. Waag, "Time-shift compensation of ultrasonic pulse focus degradation using least-mean square error estimates of arrival time," J. Acoust. Soc. Amer., vol. 95, pp. 542-555, 1994.

[5] A. P. Berkhoff, P. M. van den Berg, and J. M. Thijssen, "Iterative calculation of reflected and transmitted acoustic waves at a rough interface," IEEE Trans. Ultrason., Ferroelect., Freq. Contr., vol. 42, pp. $663-671,1995$

[6] _ _ "Simulation of wave propagation through aberrating layers of biological media," in 1994 IEEE Ultrason. Symp. Proc., Cannes, France, Nov. 1-4, vol. 3, pp. 1797-1800, 1994.

[7] D. P. Orofino and P. C. Pedersen, "Efficient angular spectrum decomposition of acoustic sources. Part I: Theory; Part II: Results," IEEE Trans. Ultrason., Ferroelect., Freq. Contr., vol. 40, pp. 238-257, 1993.

[8] E. G. Williams and J. D. Maynard, "Numerical evaluation of the Rayleigh integral for planar radiators using the FFT," J. Acoust. Soc. Amer., vol. 72, pp. 2020-2030, 1982.

[9] A. P. Berkhoff, J. M. Thijssen, and R. J. F. Hornan, "Simulation of ultrasonic imaging with linear arrays in causal absorptive media," Ultrasound Med. Biol., in press.

[10] G. R. Lockwood, J. W. Hunt, and F. S. Foster, "The design of protection circuitry for high-frequency ultrasound imaging systems," IEEE Trans. Ultrason., Ferroelect., Freq. Contr., vol. 38, pp. 48-55, 1991.

[11] P. Altmeyer, S. el-Gammal, and K. Hoffmann, Ultrasound in Dermatology. Berlin: Springer-Verlag, 1992.

[12] A. J. Berkhout, Applied Seismic Wave Theory. Ansterdam: Elsevier, 1987.

[13] J. C. Lockwood and J. G. Willette, "High-speed method for computing the exact solution for the pressure variations in the nearfield of a baffied piston," J. Acoust. Soc. Amer., vol. 53, pp. 735-741, 1973.

[14] P. M. van den Berg, "Tterative computational techniques in scattering based upon the integrated square error criterion," IEEE Trans. Antennas Propagat., vol. AP-32, pp. 1063-1071, 1984.

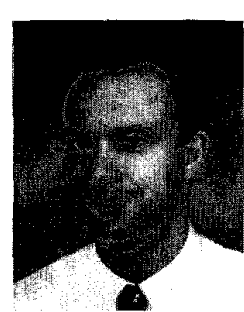

Léon A. F. Ledoux was born in Heerlen, The Netherlands on May 1, 1971. He received the M.Eng.Sc. degree in electrotechnical engineering at Eindhoven University of Technology in 1995. He worked at the Laboratory of Biophysics from October 1993 until February 1995 while working on the M.Eng.Sc. degree thesis.

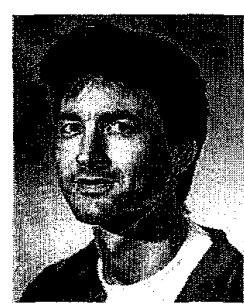

Arthur P. Berkhoff (S'94) was born in Wisch, The Netherlands on June 28, 1964. He received the B.Sc. degree in electrical engineering from the Enschede Polytechnic in 1987. After a year of military service, he studied electrical engineering at the University of Twente, Enschede, where he received the M.Sc. degree in 1991.

Currently, he is working as a Research Engineer at the University Hospital, Nijmegen, where he is engaged in research on computational models for ultrasonic wave propagation in human tissue. Mr. Berkhoff is a member of the Audio Engineering Society.

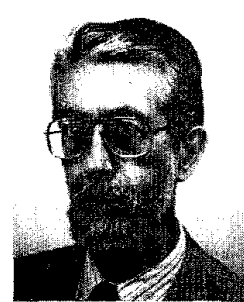

Johan M. Thijssen (A'93-M'93-SM'94) was born in Waalwijk, The Netherlands on May 12, 1939. He received the M.Eng.Sc. degree in technological physics from the Technological University of Delft, The Netherlands in 1964, and the Ph.D. degree in experimental physics from the University of Nijmegen, The Netherlands in 1969. His Ph.D. dissertation was devoted to the differential luminance sensitivity of the human visual system.

From 1964 until 1968, he was a Research Assistant in the Department of Medical Physics at the University of Nijmegen. He continued his interests in the field of differential luminance sensitivity of the human visual system with microelectrophysiological studies of the visual system of animals until 1980. In 1968, he became Head of the Biophysics Laboratory of the Department of Ophthalmology, University of Nijmegen. In 1969, he started instrumental developments for ophthalmological echography, an immersion compound scanner, and more recently, he patented a mechanical real-time scanner. In 1975, he received his first grant for research in the field of ultrasonic tissue characterization; this research is continuing. In 1980, he organized the 8th Congress of the International Society for Ultrasound in Ophthalmology. From 1982 until 1988 , he was the Project Leader of a Concerted Action Programme on Ultrasonic Tissue Characterization of the European Communities. Between 1979 and 1993, he organized nine European workshops on this topic.

Dr. Thijssen is a member of the National Health Council and National Delegate in the European and World Federations for Ultrasound in Medicine and Biology, and in the International Organization for Medical Physics. He is Cofounder and past President of The Netherlands Society for Ultrasound in Medicine and Biology, and past Secretary/Treasurer of the International Society for Ultrasound in Ophthalmology. He is the founder (1993) and Editor-in-Chief of the European Journal of Ultrasound. 